\title{
UPGRADING OF THE LICK-GAERTNER AUTOMATIC MEASURING SYSTEM
}

\author{
L. B. ROBINSON and S. VASILEVSK IS \\ Lick Observatory, Santa Cruz, Calif., U.S.A.
}

\begin{abstract}
The Automatic Measuring System (Lick Obs. Bull., No. 598, 1971) was built according to a design developed in 1959. Technological advances since then, particularly in small computers and in electronic component reliability, offered an opportunity for a substantial upgrading of the system. Most of the electronic circuitry has been replaced using modern components, and a PDP- 8 computer has been added to the system. Punched card equipment has been replaced by magnetic tapes.
\end{abstract}

The Lick Automatic Measuring System was built by the Gaertner Scientific Corporation, Chicago, in accordance with a design formulated in 1959, and it was installed at the Santa Cruz laboratories of Lick Observatory in 1967. This paper describes work done recently to upgrade the performance of the machine; not so much to increase accuracy of measurement, which has always been satisfactory, but to improve the convenience of operation, increase the rate at which measurements can be done, and to reduce the amount of human supervision required for its normal operation.

The System consists of two components: the Survey Machine and the Automatic Measuring Engine. Since details of construction and past operation are given elsewhere (Lick Obs. Bull., No. 598. 1971), only a general outline will be offered here.

The Survey Machine is used for initial inspection of plates and selection of objects for subsequent measurement. Two plates up to $17 \times 17$ in. each can be surveyed simultaneously by means of projection on a screen. Originally, approximate coordinates of selected objects were measured and recorded on punched cards in this process of surveying. These cards then served as input for the automatic measuring process. A card was read, the Automatic Engine located the object, and then photometric and precise coordinate measurements were made and punched on a card. Normally this process was automatically repeated until the last card of the survey deck was read.

The Automatic Measuring System has been heavily used, with a remarkable increase in precision and efficiency over traditional methods. There have occurred, however, many interruptions in the automatic process, caused by occasionally insufficient accuracy of input coordinates, by errors and failures in the card reader and punch, in the relays which controlled the measuring sequence, and by occasional mechanical malfunctions. The number of interruptions has been large enough that unattended operation of the machine was not feasible. The attendant had such tasks as manual centering of poorly located stars, clearing the card punch or reader in case of a malfunction, and restarting the operation in cases when no star was found, or when the servo system could not center an image for some reason. These interruptions were frequent enough to cause us to consider an improvement of the machine by utilizing technological advances that have occurred since it was designed and built.

Originally we considered merely replacing the most troublesome relay circuitry by 
solid state electronics and adding more electronic controls to reduce the need for operator supervision. However, modifications to a special purpose control system often produce unintended results that require more modifications. Such patchwork changes are expensive in engineering time and are also liable to cause difficulties in maintenance. Furthermore, future changes and improvements would be equally expensive and difficult. On the other hand, the use of an on-line computer as a controller means that future improvements in the control logic and in the operating procedure can be made and tested by the user, without the need of engineering support, and with full assurance that a change which proves undesirable can be eliminated by just going back to a previous program. It is also true that a computer, because it is a mass-produced and thoroughly tested instrument, should give much better reliability than could be expected of any one-of-kind special control circuitry. Consequently, the decision was made to use a small computer to provide the additional control needed for the Automatic Measuring System.

During the past winter, the control system for the Measuring Engine has been completely redesigned and rebuilt. The work has been done in two stages. We first replaced the relay controls by solid state circuitry, thus eliminating a major source of interruptions and trouble calls. The machine was then operated normally for several months to test the new components. In June we removed the card reading and punch equipment, and associated electronics, replacing these with a PDP-8 computer, plus magnetic tapes. The computer was also connected to the new solid-state circuitry so that it can monitor and supervise each step of the measuring process.

The second stage of the upgrading project is almost complete. The computer can now automatically correct for plate misalignment, rapidly locate stars on the plate and control the measuring process. A primitive data acquisition program is in operation. However, we have not yet completed the rather large programming job to operate the system efficiently. Also, detailed tests of measurement precision with the new system have yet to be carried out.

The third stage of upgrading will be to connect the manual Survey Machine to the computer instead of the present card punch. This will require only minor hardware additions. Some careful programming will be required to allow the survey and measuring operation to use the computer simultaneously without wasting time for either of these operations.

When the upgrading is done, the PDP-8 will control all data acquisition, data formatting and storage on magnetic tape. Reduction of final output data will continue to be carried out at the campus computer center using existing programs, with minor changes because the input is now from magnetic tape rather than punched cards.

It is well known that programming a small computer for on-line work can be a very big job. We believe, however, that it will be possible to prepare the necessary programs to operate the Automatic Measuring System without an undue expenditure of programming time. This is partly because Lick Observatory has already installed two other identical PDP-8 computers for other applications, and much of the existing proven software can be used in this new project. 
The FOCAL programming language (developed by Digital Equipment Corporation) has been chosen for this work; it is very similar to the BASIC language and is easily mastered. The floating point arithmetic with 10 digit precision is rather slow, but more than fast enough for our needs, where the main time delay occurs while waiting for mechanical motions in the Measuring Engine. At Lick Observatory, we have added a large number of additional commands, so that hardware devices such as the Automatic Measuring System are controlled by simple program statements which are easily learned and used. Program sequences using the commands are prepared by typing on a teletype and may be immediately tested, used and stored.

Programs are stored on a miniature magnetic tape (DECTAPE) which is fully addressable and on which individual segments of information can be written without disturbing other data. Programs are written, stored on tape, recalled, edited and stored again as often as desired. No compiling is necessary because the language is an interpretive one. Programs can link together, one program calling another as a subroutine from tape. A program is typically equivalent in computing power to about a page of FORTRAN coding. Over a hundred such programs can be linked together on one magnetic tape, so that even with our small computer we can prepare and use very large and complex program sequences.

It is expected that the upgrading will be completed before the end of this year (1973), with a resulting increase in precision, reliability and efficiency. While we hesitate to speculate on the increase of precision, before thorough tests are carried out, the reliability and efficiency can be discussed from the experience and from the design, based on known facts about operation with on-line computers.

As mentioned, the troublesome relays have been replaced by tested and reliable solid-state circuitry. Furthermore, the original hard-wired unit for control of machine operations, and the mechanical readers and punches with associated electronics have been replaced by the PDP- 8 computer, again a tested and reliable piece of equipment. These changes should greatly reduce the frequency of service calls to take care of malfunctions. In case of a malfunction, however, the computer should permit easy diagnosis of the cause.

The increase in reliability will be one but not the only reason for increased efficiency. As mentioned at the outset, two plates of the same field can be surveyed simultaneously. Only one plate, located in the fixed plate adapter whose coordinate reference corresponds to that of the Automatic Measuring Engine, could be measured automatically without further adjustments. For automatic measurement of the other plate either a mechanical alignment had to be made or a new input deck had to be produced by the campus computer from initial manual measurement of three or more control stars. The need of adjustments was especially time consuming in case many plates of the same field had to be measured, as in parallax work. Depending on number of plates or of objects to be measured, either mechanical or off-line computer adjustments were made, or the plate motion was even controlled manually when the number of objects was not large enough to justify these alignments. Using the on-line computer, of course, the mathematical adjustment of the input coordi- 
nates will be fully automatic after the operator initially identifies a few control stars.

A perfect alignment of the plate on the Measuring Engine did not guarantee automatic measurement of every object. Occasionally insufficient care in centering while surveying, or large proper motion may lead to positioning the object completely outside the area of the spinning-sector scanner. In the past this would interrupt the automatic measuring sequence. Now the effect of approximately known proper motion can be taken care of by the computer. If the object is not found for other reasons, an automatic search will be carried out, and this fact will be recorded on the teletype. Regardless of finding or not finding the object, the automatic operation will not stop. When one or more subsequent plates of the same field are measured, the photometric and coordinate measurements will be compared with those of the previous plate, and if the discrepancy exceeds a chosen limit, the fact will again be recorded by the teletype, without interrupting the automatic sequence. Thus the operator can leave the Automatic Engine alone after starting it, and then return only to inspect the causes of typed messages. Before the plate is removed, suspicious cases can be rechecked while the operator watches the operation with a closed circuit television monitor. The computer program could also remeasure suspicious cases before the operator returns. The typed messages and automatic error checking should greatly reduce the editing work which in the past was a time-consuming task in detecting mispunches and spurious measurements.

Finally, even mechanical motions can be made more efficient. In the past there was one slewing and two setting speeds for the heavy plate adapter. In order to prevent unacceptable jolts in switching from one to another speed, the range of speeds had to be quite limited, but now the computer can cause continuous acceleration and deceleration.

The above mentioned reasons for increased efficiency are those planned at present. The versatility of computer control and data processing may offer other improvements not visualized at present but which may become obvious and feasible with experience gained.

\section{Acknowledgement}

The project of upgrading is being supported by the National Science Foundation, Grant GP-32459.

\section{DISCUSSION}

Wall: Does the machine introduce a magnitude equation, and if so is it removed by the computer programme?

Vasilevskis: Yes, a magnitude equation is introduced, and it is not removed yet by the computer. We may do it in future, however. In our astronomical programme the magnitude effect is nearly eliminated, because

(1) We are interested in differential measurements of nearly identical plates, and

(2) An objective grating is used, and grating images (1st or 2 nd order spectra) are measured for bright stars. 